\title{
Bulk Synaptic Vesicle Endocytosis Is Rapidly Triggered during Strong Stimulation
}

\author{
Emma L. Clayton, ${ }^{1}$ Gareth J. O. Evans, ${ }^{2}$ and Michael A. Cousin ${ }^{1}$ \\ ${ }^{1}$ Membrane Biology Group, Centre for Integrative Physiology, George Square, University of Edinburgh, Edinburgh EH8 9XD, United Kingdom, and \\ ${ }_{2}^{2}$ Department of Biology (Area 3), University of York, York Y010 5YW, United Kingdom
}

\begin{abstract}
Bulk endocytosis in central nerve terminals is activated by strong stimulation; however, the speed at which it is initiated and for how long it persists is still a matter of debate. To resolve this issue, we performed a characterization of bulk endocytic retrieval using action potential trains of increasing intensity. Bulk endocytosis was monitored by the loading of the fluorescent dyes FM2-10 and FM1-43, uptake of tetramethylrhodamine-dextran $(40 \mathrm{kDa})$, or morphological analysis of uptake of the fluid-phase marker horseradish peroxidase. When neuronal cultures were subjected to mild stimulation (200 action potentials at $10 \mathrm{~Hz})$, bulk endocytosis was not observed using any of our assay systems. However, when more intense trains of action potentials (400 or 800 action potentials at $40 \mathrm{and} 80 \mathrm{~Hz}$, respectively) were applied to neurons, bulk endocytosis was activated immediately, with the majority of bulk endocytosis complete by the end of stimulation. This contrasts with single synaptic vesicle endocytosis, the majority of which occurred after stimulation was terminated. Thus, bulk endocytosis is a fast event that is triggered during strong stimulation and provides the nerve terminal with an appropriate mechanism to meet the demands of synaptic vesicle retrieval during periods of intense synaptic vesicle exocytosis.
\end{abstract}

Key words: endocytosis; bulk; synaptic vesicle; nerve terminal; FM1-43; clathrin

\section{Introduction}

Endocytosis of synaptic vesicles (SVs) after exocytosis is essential for the maintenance of neurotransmission in central neurons. At least two different mechanisms retrieve SV membrane from the nerve terminal plasma membrane dependent on the intensity of stimulation. During mild stimulation, clathrin-dependent endocytosis predominates (Granseth et al., 2006), in which SVs are generated directly from the plasma membrane via a well characterized molecular mechanism involving clathrin, the adaptor proteins AP-2 and AP180, and a host of accessory proteins (Murthy and De Camilli, 2003; Royle and Lagnado, 2003). A second endocytosis pathway called bulk endocytosis is also recruited during more intense stimulation (Royle and Lagnado, 2003; Rizzoli and Betz, 2005; Wu et al., 2007). Bulk endocytosis involves the invagination of large areas of plasma membrane to form endosomal-like compartments. SV s can then bud from these endosomal intermediates and rejoin the recycling SV pool (Richards et al., 2000; Evans and Cousin, 2007). The molecules that control this pathway are still undetermined; however, the dephosphorylation and rephosphorylation of a group of endocytosis proteins called the dephosphins by the calcium-dependent protein phosphatase calcineurin and the protein kinase cyclindependent kinase 5 is required (Evans and Cousin, 2007).

Received March 7, 2008; revised May 16, 2008; accepted May 17, 2008

This work was supported by both The Wellcome Trust Grant GR070569 and Epilepsy Research UK. We thank Dr. Alan Prescott and John James (both University of Dundee, Dundee, UK) for their excellent technical assistance.

Correspondence should be addressed to Michael A. Cousin, Membrane Biology Group, Centre for Integrative

Physiology, George Square, University of Edinburgh, Edinburgh EH8 9XD, UK. E-mail: m.cousin@ed.ac.uk.

DOI:10.1523/JNEUROSCI.1445-08.2008

Copyright $\odot 2008$ Society for Neuroscience $\quad$ 0270-6474/08/286627-06\$15.00/0
Clathrin-dependent endocytosis is a relatively slow process with a limited capacity that occurs with a time constant of $\sim 15 \mathrm{~s}$ (Sankaranarayanan and Ryan, 2000; Granseth et al., 2006; Balaji and Ryan, 2007). It is proposed that during strong stimulation, the clathrin-dependent machinery becomes saturated, and bulk endocytosis is recruited to retrieve additional membrane (Royle and Lagnado, 2003; Wu et al., 2007). However, bulk endocytosis is thought to be slower than clathrin-dependent endocytosis, persisting for minutes after termination of stimulation (Richards et al., 2000; Teng and Wilkinson, 2000; Wu et al., 2007). This places the nerve terminal in a dangerous situation, because there would be no retrieval mechanism to rapidly correct the large increase in membrane surface area produced by excessive SV exocytosis.

The speed at which bulk endocytosis occurs and for how long it persists is still matter of debate (Wu et al., 2007); however, both aspects are key to understanding how central nerve terminals cope with strong stimuli. To clarify this question, we characterized bulk endocytosis in central nerve terminals using a range of stimulation paradigms and a number of different assay systems. We found that bulk endocytosis was a fast process that was triggered by strong physiological stimulation and was predominantly complete by termination of stimulation. Thus, bulk endocytosis provides the nerve terminal with the appropriate membrane retrieval options to cope with large stimulation loads over a short period of time.

\section{Materials and Methods}

Materials. FM2-10, FM1-43, tetramethylrhodamine-dextran, penicillin/ streptomycin, phosphate-buffered salts, fetal calf serum, and minimal essential medium were obtained from Invitrogen. Glutaraldehyde and 
osmium tetroxide were from Agar Scientific. All other reagents were from Sigma.

Fluorescence imaging of SV turnover. Primary cultures of cerebellar granule neurons were prepared from the cerebella of 7-d-old Sprague Dawley rat pups (Tan et al., 2003). Cultures were removed from culture medium and left for $10 \mathrm{~min}$ in incubation medium [170 $\mathrm{mm} \mathrm{NaCl}, 3.5$ mM KCl, $0.4 \mathrm{~mm} \mathrm{KH}_{2} \mathrm{PO}_{4}, 20 \mathrm{~mm}$ TES ( $N$-tris[hydroxy-methyl]-methyl2-aminoethane-sulfonic acid), $5 \mathrm{~mm} \mathrm{NaHCO}, 5 \mathrm{~mm}$ glucose, $1.2 \mathrm{~mm}$ $\mathrm{Na}_{2} \mathrm{SO}_{4}, 1.2 \mathrm{mM} \mathrm{MgCl}_{2}$, and $1.3 \mathrm{mM} \mathrm{CaCl}_{2}, \mathrm{pH}$ 7.4]. Cultures were then mounted in a Warner imaging chamber (RC-21BRFS). Invaginating membrane was loaded with either FM2-10 $(100 \mu \mathrm{M})$ or FM1-43 $(10 \mu \mathrm{M})$ by evoking SV turnover using trains of action potentials $(100 \mathrm{~mA}, 1 \mathrm{~ms}$ pulse width). Dye was washed from the cultures immediately after termination of stimulation with incubation medium (Fig. $1 \mathrm{~A}$ ). After a 10 min rest period, accumulated dye was unloaded from nerve terminals using two sequential $30 \mathrm{~s}$ stimuli of either $50 \mathrm{~mm} \mathrm{KCl}$ or 400 action potentials (at $40 \mathrm{~Hz}$ ) at 20 and $120 \mathrm{~s}$. This provides an estimate of the total number of SVs turned over during stimulation under control conditions $(\Delta \mathrm{S} 1)$. After a $20 \mathrm{~min}$ rest period, this protocol was repeated, only this time washout of dye was delayed by $5 \mathrm{~min}(\Delta \mathrm{S} 2)$. Dye unloading was visualized using a Nikon epifluorescence microscope (Diaphot-TMD) and $20 \times$ air objective at $480 \mathrm{~nm}$ excitation and $>510 \mathrm{~nm}$ emission. Fluorescent images were acquired with a Hamamatsu Orca-ER CCD digital camera and off-line imaging software (Simple PCI; Compix Imaging Systems). $\Delta \mathrm{S} 2 / \Delta \mathrm{S} 1$ ratios from individual experiments (at least 30 nerve terminals per experiment) for the same condition were averaged. Cultures were used routinely between 8 and $10 \mathrm{~d}$ in vitro.

Fluorescence imaging of dextran uptake. Cultures were removed from culture medium and left for $10 \mathrm{~min}$ in incubation medium. Tetramethylrhodamine-dextran $(40 \mathrm{kDa})$ was applied either during trains of action potentials or immediately afterward for $2 \mathrm{~min}$. Dextran was washed from the cultures, and the extent of loading was examined using a $20 \times$ air objective at $550 \mathrm{~nm}$ excitation and $>575 \mathrm{~nm}$ emission. The extent of dextran loading was determined by the number of fluorescent puncta in a defined field of view $(130 \times 130 \mu \mathrm{m})$. Thresholding analysis was performed to discount regions too large to represent individual nerve terminals (diameter $>2$ $\mu \mathrm{m})$. Dextran puncta colocalized with nerve terminals as assessed by coloading with FM1-43 (data not shown). The average fluorescence intensity of individual puncta did not vary significantly between different stimulation conditions (data not shown). The average number of dextran puncta per field for each experiment (usually 10 fields of view per experiment) were averaged for the same conditions. Cultures were used routinely between 8 and $10 \mathrm{~d}$ in vitro.

Labeling of endocytic pathways by horseradish peroxidase. Cultures were processed for electron microscopy as described previously (Deak et al., 2004; Evans and Cousin, 2007) with minor modifications. Cultures were removed from culture medium into incubation medium, and after a 10 min rest period, cultures were stimulated with trains of action potentials. Medium was supplemented with horseradish peroxidase (HRP; $10 \mathrm{mg}$ / $\mathrm{ml}$ ) either during stimulation or for $5 \mathrm{~min}$ after stimulation. Neurons were fixed in a $2 \%$ solution of glutaraldehyde in PBS for $30 \mathrm{~min}$ at $37^{\circ} \mathrm{C}$. After washing with $100 \mathrm{~mm}$ Tris ( $\mathrm{pH} 7.4$ ), cultures were exposed to $0.1 \%$ diaminobenzidine and $0.2 \% \mathrm{H}_{2} \mathrm{O}_{2}$ in $100 \mathrm{~mm}$ Tris. On development of color, cultures were washed with $100 \mathrm{~mm}$ Tris and then stained with $1 \%$ osmium tetroxide for $30 \mathrm{~min}$. After washing, cultures were poststained with $2 \%$ uranyl acetate for $15 \mathrm{~min}$ and then dehydrated using ethanol series and polypropylene oxide and embedded using Durcupan. Samples were sectioned, mounted on grids, and viewed using an FEI Tecnai 12 transmission electron microscope. Nerve terminals that contained HRP were analyzed, and intracellular structures that were $<100 \mathrm{~nm}$ in diameter were arbitrarily designated to be SVs, whereas larger structures were designated to be endosomes. Data from individual nerve terminals for the same condition were averaged and tested for statistical significance.

\section{Results}

\section{Delayed addition of FM dyes does not differentially label} bulk endocytosis

Previous studies have shown a differential labeling of bulk endocytosis with the dyes FM1-43 and FM2-10 (Richards et al., 2000;
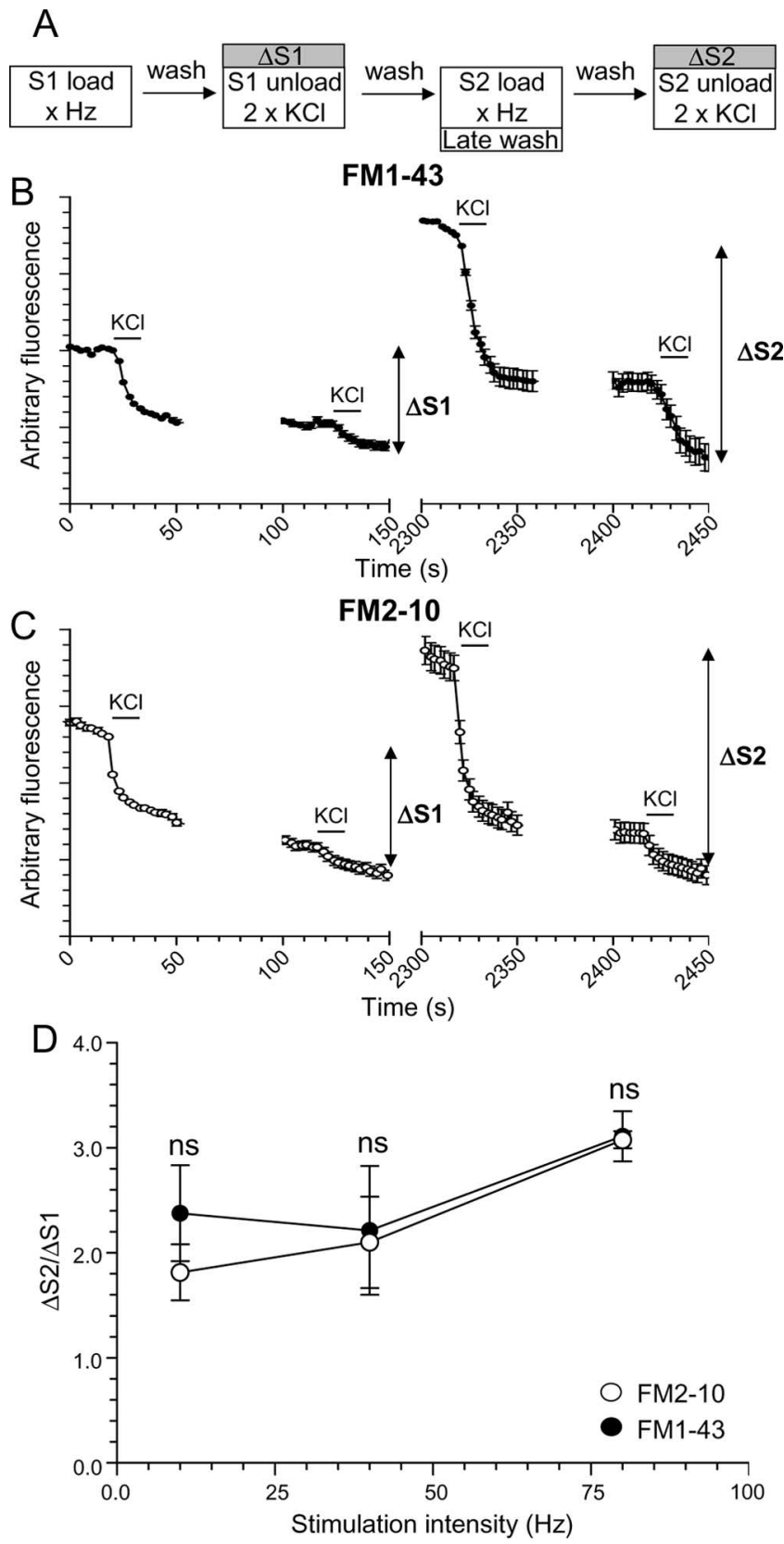

Figure 1. No disparity in action potential-evoked poststimulation loading for FM1-43 and FM2-10. $A$, Granule neuron cultures were loaded with either FM1-43 or FM2-10 using trains of $200(10 \mathrm{~Hz}), 400(40 \mathrm{~Hz})$, or $800(80 \mathrm{~Hz})$ action potentials. Dyes were loaded during stimulation at $\mathrm{S1}$, but washout was delayed for $5 \mathrm{~min}$ at $\mathrm{S2}$. Unloading was stimulated by 30 s stimuli of 50 тм KCl except for cultures loaded with 200 action potentials, for which two sequential trains of 400 action potentials $(40 \mathrm{~Hz})$ were used. $\boldsymbol{B}, \boldsymbol{C}$, Time course of the average response for nerve terminals loaded with either FM1-43 (B) or FM2-10 ( $C$ ) during action potential stimulation at 80 $\mathrm{Hz}$ is displayed. $\boldsymbol{D}$, Mean $\Delta S 2 / \Delta S 1$ ratio \pm SEM is displayed for each stimulation condition. All experiments were performed four times except those with 200 action potentials $(n=3)$. No significant difference between FM2-10 and FM1-43 loading was observed during any stimulation protocol (Student's $t$ test).

Virmani et al., 2003). The reason proposed for this was that after its washout, the more hydrophobic FM1-43 could label large endocytic structures that were retrieved after termination of stimulation, whereas the more hydrophilic FM2-10 could not. However, if FM2-10 washout was delayed, labeling of poststimulation bulk endocytosis was observed (Richards et al., 2000).

We monitored FM1-43 and FM2-10 labeling of endocytosis 
pathways that occurred both during and after action potential trains of increasing intensity. Neuronal cultures were subjected to two rounds of dye loading and unloading, with extent of membrane turnover during each stimulus quantified by monitoring the amount of dye unloading from individual nerve terminals ( $\Delta \mathrm{S} 1$ and $\Delta \mathrm{S} 2$ ) (Fig. $1 A$ ). The extent of dye loading was compared between an immediate washout of dye at $\mathrm{S} 1$ or a delayed (5 min) washout after termination of stimulation at S2. Thus, the proportion of membrane retrieving via bulk endocytosis after stimulation should be assessed by comparing FM2-10 loading to FM143. As a control, cultures were first challenged with trains of 200 action potentials $(10 \mathrm{~Hz})$, a protocol that does not recruit bulk endocytosis (Evans and Cousin, 2007). Approximately twice as much loading occurred at S2 (delayed washout) for both dyes, compared with S1 (immediate washout) (Fig. 1D). As expected, no additional loading of FM2-10 was observed (Fig. 1D). Thus, the poststimulation membrane retrieval pathway during mild stimulation is likely to be single SV endocytosis, because (1) bulk endocytosis is not activated with this stimulation protocol, and (2) there is no additional FM2-10 loading compared with FM1-43.

Cultures were then challenged with more intense stimuli of either $400(40 \mathrm{~Hz})$ or $800(80 \mathrm{~Hz})$ action potentials, to recruit bulk endocytosis in addition to the single SV route. Much greater poststimulation uptake was observed for both FM1-43 and FM210 , indicating that more endocytosis was occurring, probably because of the increased stimulus load (Fig. 1D). However, no disparity in loading was observed between FM2-10 and FM1-43. This suggests that either (1) no bulk endocytosis is activated by strong physiological stimulation, or (2) bulk endocytosis is immediately triggered during stimulation and is predominantly complete even after brief action potential trains.

\section{Bulk endocytosis is triggered immediately during strong action potential stimulation}

To address these possibilities, a more direct measure of bulk endocytosis was performed, namely the uptake of a large $(40 \mathrm{kDa})$ fluorescent dextran (tetramethylrhodamine-dextran). These dextrans are too large to be accumulated by single SV endocytosis and are more selective markers of fluid-phase bulk uptake (Holt et al., 2003; Teng et al., 2007). Incubation of cultures with fluorescent dextran during 200 action potentials resulted in no apparent uptake (Fig. $2 A, G$ ), confirming that mild stimulation does not recruit bulk endocytosis. In contrast, when cultures were stimulated with either 400 or 800 action potentials, a large uptake of dextran was observed during the $10 \mathrm{~s}$ train (Fig. $2 C, E, G)$. Thus, bulk endocytosis does occur in response to physiological trains of action potentials, because the single SV route cannot internalize large dextrans (Fig. $2 \mathrm{~A}$ ). When internalization of dextran was assessed after stimulation, little uptake was observed over background levels for any condition (Fig. 2D,F,H). This suggests that bulk endocytosis is triggered immediately by brief trains of action potentials and does not persist to any great extent after stimulation.

To confirm this statement, experiments were performed with the fluid-phase marker HRP. This has the advantage that structures (SVs or bulk endosomes) that are directly retrieved from the plasma membrane can be visualized at the level of a single nerve terminal. When cultures were challenged with a mild stimulus of 200 action potentials, very little uptake into endosomes was observed either during or after stimulation (Fig. $3 G, H$ ), again demonstrating that bulk endocytosis is not activated by mild stimulation. However, when HRP was applied during trains of 400 and
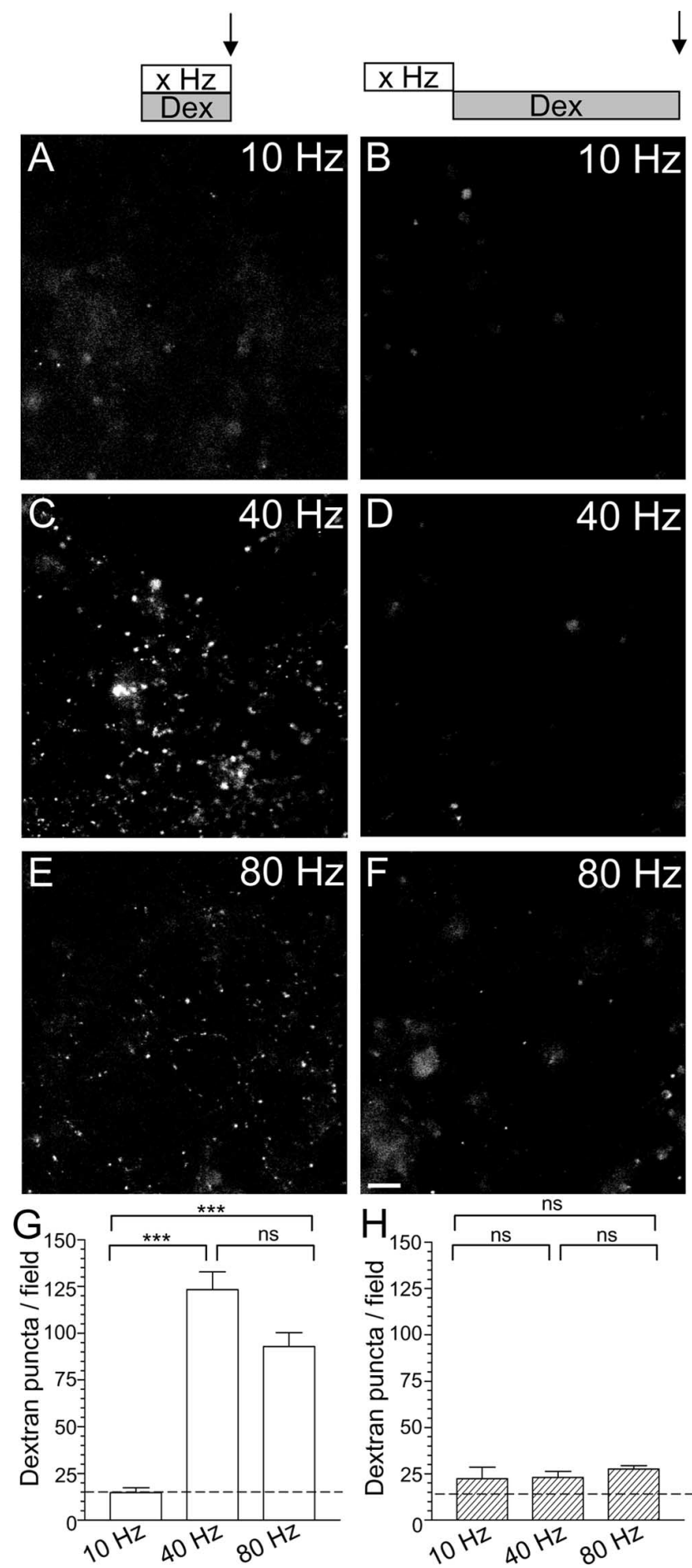

Figure 2. Uptake of large dextrans only occurs during strong action potential stimulation. Left, Representative fields in which tetramethylrhodamine-dextran $(50 \mu \mathrm{M})$ was applied to cultures during trains of $200(\boldsymbol{A} ; 10 \mathrm{~Hz}), 400(\boldsymbol{C} ; 40 \mathrm{~Hz})$, or $800(\boldsymbol{E} ; 80 \mathrm{~Hz})$ action potentials and then immediately washed away. Right, Fields in which dextran was applied to cultures for $2 \mathrm{~min}$ immediately after trains of $200(\boldsymbol{B} ; 10 \mathrm{~Hz}), 400(\boldsymbol{D} ; 40 \mathrm{~Hz})$, or $800(\boldsymbol{F} ; 80 \mathrm{~Hz})$ action potentials. Scale bar, $15 \mu \mathrm{m}$. Mean number of dextran puncta per field \pm SEM is displayed for either during ( $\mathbf{G}$; open bars) or after ( $\boldsymbol{H}$; hatched bars) stimulation. Dotted line illustrates puncta number per field attributable to intrinsic background fluorescence in the absence of dextran. All experiments were performed three times. ${ }^{* *} p<0.001$, one-way ANOVA.

800 action potentials, large numbers of HRP-labeled endosomes were observed (Fig. 3G,H). This number fell dramatically when HRP was applied after strong stimulation, confirming that the majority of bulk endocytosis occurred during stimulation 

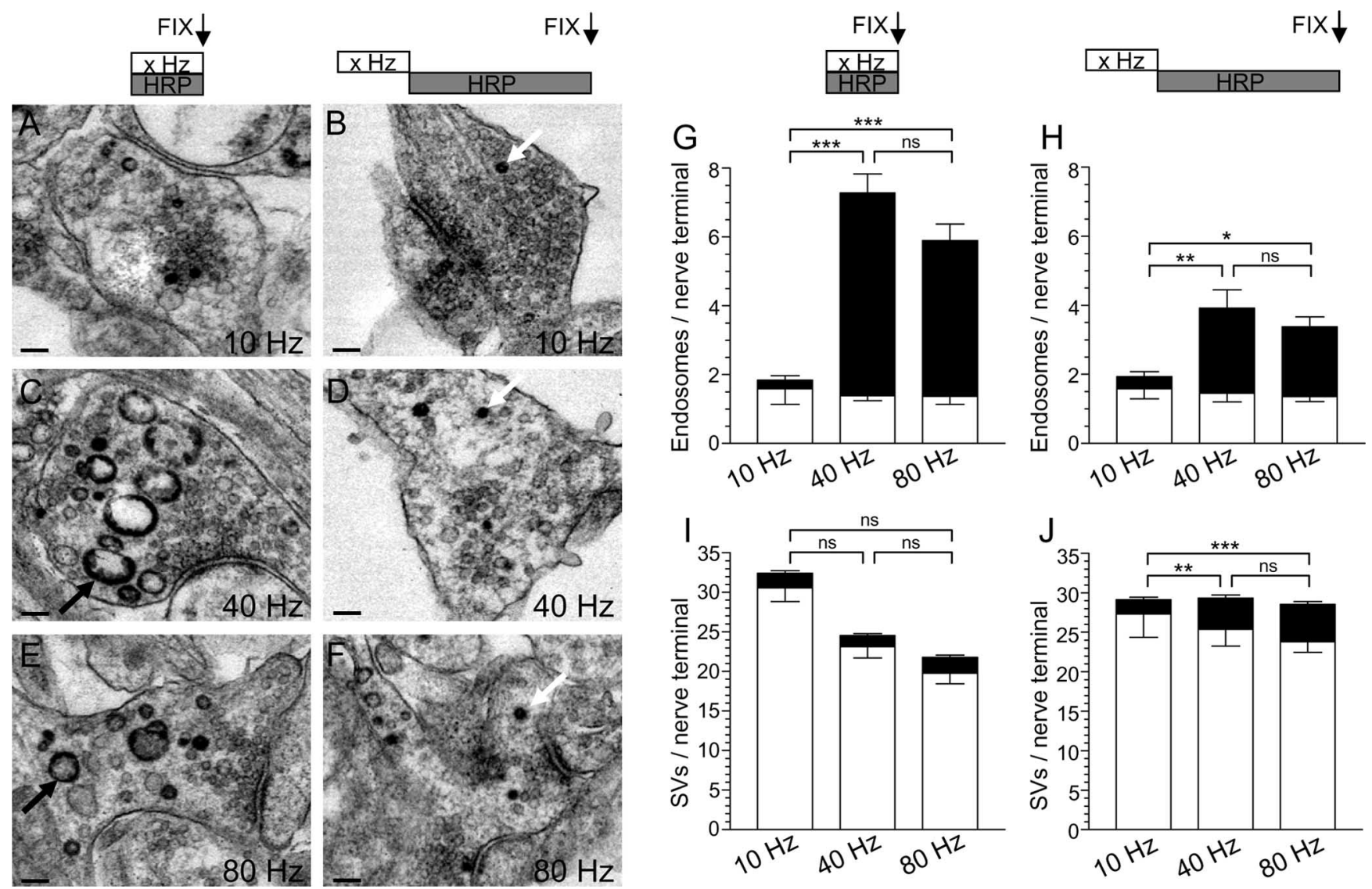

Figure 3. HRP labels bulk endosomes when applied during strong action potential stimulation. $\boldsymbol{A}-\boldsymbol{F}$, HRP was applied to cultures during trains of $200(\boldsymbol{A} ; 10 \mathrm{~Hz}), 400(\boldsymbol{C} ; 40 \mathrm{~Hz}), 0$ or $800(\boldsymbol{E} ; 80 \mathrm{~Hz})$ action potentials and then immediately fixed. Alternatively, HRP was applied to cultures for 5 min immediately after trains of $200(\boldsymbol{B} ; 10 \mathrm{~Hz}), 400(\boldsymbol{D} ; 40 \mathrm{~Hz})$, or $800(\boldsymbol{F} ; 80 \mathrm{~Hz})$ action potentials and then fixed. Representative electron micrographs are displayed. Black arrows indicate HRP-labeled endosomal structures, whereas white arrows indicate HRP-labeled SVs. Scale bars, $100 \mathrm{~nm}$. Mean number \pm SEM of either HRP-labeled (solid bars) or clear (open bars) endosomes (G) or SVs $(\boldsymbol{I})$ generated during stimulation is displayed (200 action potentials, $n=17$ nerve terminals; $400, n=$ $26 ; 800, n=53$ ). Mean number \pm SEM of either HRP-labeled (solid bars) or clear (open bars) endosomes $(\boldsymbol{H})$ or SVs $(\boldsymbol{J})$ generated after stimulation is displayed per nerve terminal (200 action potentials, $n=18$ nerve terminals; $400, n=39 ; 800, n=38) .{ }^{*} p<0.05,{ }^{* *} p<0.01,{ }^{* * *} p<0.001$, one-way ANOVA for HRP structures.

( $\sim 70 \%$ during stimulation for both 400 and 800 action potentials) (Fig. $3 G, H$ ). Thus, strong physiological stimulation immediately triggers bulk endocytosis to cope with additional membrane retrieval demands.

When HRP labeling of SVs was monitored, a different picture emerged. During mild stimulation with 200 action potentials, an increase in HRP-labeled SVs was observed both during and after stimulation (Fig. $3 I, J$ ), showing that the single SV route is activated by mild stimulation and that it persists after stimulation (Evans and Cousin, 2007). However, unlike bulk endocytosis, the number of HRP-labeled SVs that were generated during stimulation did not increase with increasing stimulation intensity, indicating that the single SV route may have reached its maximum capacity (Sankaranarayanan and Ryan, 2000) (Fig. 3I,J). There was a large increase in the number of HRP-labeled SVs after stimulation, with either 75 or $72 \%$ of all HRP SVs retrieved after termination of stimulation with either 400 or 800 action potentials (Fig. $3 I, J$ ). Thus, the major membrane retrieval pathway that persists after termination of strong stimulation is the single SV route, and not bulk endocytosis.

\section{Discussion}

Bulk endocytosis in central nerve terminals is activated by strong stimulation protocols and has been proposed to occur slowly, persisting after stimulation has been terminated. We demonstrate that bulk endocytosis is triggered immediately during strong stimulation and that the majority of the process is complete during stimulation. Evidence for this is as follows: during trains of high frequency action potentials, (1) no disparity between FM2-10 and FM1-43 loading was observed after stimulation; (2) uptake of large dextrans was only observed when they were applied during stimulation; and (3) using HRP to label the fluid phase, bulk endocytosis was the major retrieval pathway during stimulation, whereas the major poststimulation retrieval pathway was the single SV route. Thus, the majority of bulk endocytosis occurs during strong stimulation, and the major poststimulation retrieval pathway is single SV clathrin-dependent endocytosis.

\section{Generation of bulk endosomes occurs during strong physiological stimulation}

Our data show that the term "slow endocytosis" is not an accurate description of bulk endocytosis. In fact, our data using brief action potential trains in central nerve terminals agree with early reports proposing that bulk endocytosis was immediately activated by excessive stimulation, and was complete within $10 \mathrm{~s}$ in the frog neuromuscular junction (Miller and Heuser, 1984). Other evidence that bulk endocytosis occurs with fast kinetics can be found in primary cultures of cerebellar granule neurons and isolated nerve terminals, where HRP-labeled bulk endosomes were abundant within 5-15 s of stimulation with elevated $\mathrm{KCl}$ (Marxen et al., 1999; Leenders et al., 2002). In retinal bipolar 
neurons, maximal uptake of fluorescent dextrans occurred within the first minute of continual stimulation with calcium and $\mathrm{KCl}$ (Holt et al., 2003). In the same preparation, the majority of the fluid-phase marker ferritin was accumulated into endosomes within seconds of brief depolarizations (Paillart et al., 2003). Also, large fluorescent dextrans accumulated in reptile nerve terminals within 1-2 s of stimulation (Teng et al., 2007). Finally, in electrophysiological studies in the calyx of Held, individual bulk endocytosis events occurred with subsecond kinetics, and the majority of these events were complete within $10 \mathrm{~s}$ of stimulation ( $\mathrm{Wu}$ and $\mathrm{Wu}, 2007$ ). Thus, bulk endocytosis is a fast-triggered event that is activated during stimulation.

What is also apparent is that bulk endocytosis does not persist to any great extent after stimulation. Little uptake of fluorescent dextran occurred after stimulation, and endosomal labeling with HRP was reduced between twofold and threefold after stimulation. However, in some neuronal systems, large cisternae have been observed after strong stimulation that are still attached to the plasma membrane by a narrow neck (Koenig and Ikeda, 1996; Takei et al., 1996; Gad et al., 1998; Richards et al., 2000; Teng and Wilkinson, 2000). Therefore, the inability of large dextrans or even possibly HRP to enter these structures may explain the apparent lack of labeling. We do not think this is the case for three major reasons: (1) there is no disparity in labeling between FM2-10 and FM1-43 after stimulation [which would be expected if such structures were still attached to the plasma membrane (Richards et al., 2000)]; (2) we see no poststimulation labeling with much smaller $(10 \mathrm{kDa})$ dextrans (E. Clayton, unpublished observations); and (3) in the hundreds of nerve terminals we have observed using electron microscopy, we have never seen such cisternae in our nerve terminals. Therefore, we propose that the major poststimulation membrane retrieval pathway is the single SV route and not bulk endocytosis. This is because this pathway can be labeled with FM dyes but not large dextrans, and, importantly, the number of HRP-labeled SVs increased between twofold and threefold (whereas HRP-labeled endosomes decreased by the same factor) after stimulation. These SVs are generated directly from the plasma membrane and not from bulk endosomes, because there is no correlation between the number of HRP endosomes and SVs in nerve terminals at which HRP addition was delayed (Pearson's coefficient $r^{2}=0.0004 ; n=48$ nerve terminals; 40 and $80 \mathrm{~Hz}$ ). In agreement, clathrin-dependent endocytosis acts as the slow phase of membrane retrieval in retinal bipolar neurons, with a time constant similar to that observed in small central nerve terminals (Jockusch et al., 2005).

Because some bulk endocytosis occurs after stimulation, at first glance it appears strange that we did not observe an additional component of loading with FM2-10 after stimulation (Fig. 1). However, the reason is relatively straightforward. In the FM experiments, the dye was applied during the stimulus at S1 and then both during and after the stimulus at S2. Thus, the S2 response reports uptake from both SV and bulk endocytosis pathways during stimulation, in addition to uptake occurring after stimulation. It is therefore difficult to resolve the small component of poststimulation bulk endocytosis for two major reasons: (1) because the majority of bulk endocytosis occurs during stimulation, the poststimulation component will only be observed as a small increase in response; and (2) the amount of uptake by bulk endocytosis after stimulation is further diluted by the threefold increase in SV endocytosis that occurs after stimulation (because FM dyes label both pathways). Therefore, it is perfectly understandable that this small component of bulk endocytosis is lost in the "noise" of the assay. For this reason, we decided to examine bulk endocytosis in isolation using specific assays to visualize uptake of large fluorescent dextrans and HRP into individual nerve terminals.

\section{Bulk endocytosis is fast and is activity dependent}

The results presented provide a clear picture of the endocytosis pathways that are active in central nerve terminals both during and after different patterns of stimulation. During mild stimulation, single SV clathrin-dependent endocytosis is sufficient to cope with the demand for membrane retrieval and is the major membrane retrieval mechanism in the nerve terminal (Granseth et al., 2006). However, when nerve terminals are challenged with more intense stimulation, bulk endocytosis is recruited in parallel with the clathrin-dependent route. This provides a fast and efficient mechanism to maintain nerve terminal surface area and to retrieve SV proteins that have been inserted into the plasma membrane. We propose bulk endocytosis is not triggered by saturation of the clathrin machinery, but rather by an activitydependent increase in calcium. This increase results in activation of calcineurin and the dephosphorylation of the dephosphin set of endocytosis proteins (Evans and Cousin, 2007). Calcineurin is the perfect candidate for the activity-dependent trigger for bulk endocytosis, because it is inactive during mild stimulation and is only activated by stimulation intensities that evoke bulk endocytosis (M. A. Cousin, unpublished observations). The proposal that calcineurin is the trigger for bulk endocytosis provides the explanation as to why bulk endocytosis does not persist after stimulation, because as the intracellular calcium signal falls, calcineurin will be inactivated and its substrates rephosphorylated. Because clathrin-dependent endocytosis can proceed independent of calcineurin activity, it can persist after stimulation to recover remaining SV proteins from the plasma membrane.

\section{References}

Balaji J, Ryan TA (2007) Single-vesicle imaging reveals that synaptic vesicle exocytosis and endocytosis are coupled by a single stochastic mode. Proc Natl Acad Sci USA 104:20576-20581.

Deak F, Schoch S, Liu X, Sudhof TC, Kavalali ET (2004) Synaptobrevin is essential for fast synaptic-vesicle endocytosis. Nat Cell Biol 6:1102-1108.

Evans GJ, Cousin MA (2007) Activity-dependent control of slow synaptic vesicle endocytosis by cyclin-dependent kinase 5. J Neurosci 27:401-411.

Gad H, Low P, Zotova E, Brodin L, Shupliakov O (1998) Dissociation between $\mathrm{Ca}^{2+}$-triggered synaptic vesicle exocytosis and clathrin-mediated endocytosis at a central synapse. Neuron 21:607-616.

Granseth B, Odermatt B, Royle SJ, Lagnado L (2006) Clathrin-mediated endocytosis is the dominant mechanism of vesicle retrieval at hippocampal synapses. Neuron 51:773-786.

Holt M, Cooke A, Wu MM, Lagnado L (2003) Bulk membrane retrieval in the synaptic terminal of retinal bipolar cells. J Neurosci 23:1329-1339.

Jockusch WJ, Praefcke GJ, McMahon HT, Lagnado L (2005) Clathrindependent and clathrin-independent retrieval of synaptic vesicles in retinal bipolar cells. Neuron 46:869-878.

Koenig JH, Ikeda K (1996) Synaptic vesicles have two distinct recycling pathways. J Cell Biol 135:797-808.

Leenders AG, Scholten G, de Lange RP, Lopes Da Silva FH, Ghijsen WE (2002) Sequential changes in synaptic vesicle pools and endosome-like organelles during depolarization near the active zone of central nerve terminals. Neuroscience 109:195-206.

Marxen M, Volknandt W, Zimmermann H (1999) Endocytic vacuoles formed following a short pulse of $\mathrm{K}^{+}$-stimulation contain a plethora of presynaptic membrane proteins. Neuroscience 94:985-996.

Miller TM, Heuser JE (1984) Endocytosis of synaptic vesicle membrane at the frog neuromuscular junction. J Cell Biol 98:685-698.

Murthy VN, De Camilli P (2003) Cell biology of the presynaptic terminal. Annu Rev Neurosci 26:701-728.

Paillart C, Li J, Matthews G, Sterling P (2003) Endocytosis and vesicle recycling at a ribbon synapse. J Neurosci 23:4092-4099. 
Richards DA, Guatimosim C, Betz WJ (2000) Two endocytic recycling routes selectively fill two vesicle pools in frog motor nerve terminals. Neuron 27:551-559.

Rizzoli SO, Betz WJ (2005) Synaptic vesicle pools. Nat Rev Neurosci 6:57-69.

Royle SJ, Lagnado L (2003) Endocytosis at the synaptic terminal. J Physiol (Lond) 553:345-355.

Sankaranarayanan S, Ryan TA (2000) Real-time measurements of vesicleSNARE recycling in synapses of the central nervous system. Nat Cell Biol 2:197-204.

Takei K, Mundigl O, Daniell L, De Camilli P (1996) The synaptic vesicle cycle: a single vesicle budding step involving clathrin and dynamin. J Cell Biol 133:1237-1250.

Tan TC, Valova VA, Malladi CS, Graham ME, Berven LA, Jupp OJ, Hansra G, McClure SJ, Sarcevic B, Boadle RA, Larsen MR, Cousin MA, Robinson PJ
(2003) Cdk5 is essential for synaptic vesicle endocytosis. Nat Cell Biol 5:701-710.

Teng H, Wilkinson RS (2000) Clathrin-mediated endocytosis near active zones in snake motor boutons. J Neurosci 20:7986-7993.

Teng H, Lin MY, Wilkinson RS (2007) Macroendocytosis and endosome processing in snake motor boutons. J Physiol (Lond) 582:243-262.

Virmani T, Han W, Liu X, Sudhof TC, Kavalali ET (2003) Synaptotagmin 7 splice variants differentially regulate synaptic vesicle recycling. EMBO J 22:5347-5357.

Wu L-G, Ryan TA, Lagnado L (2007) Modes of vesicle retrieval at ribbon synapses, calyx-type synapses, and small central synapses. J Neurosci 27:11793-11802.

Wu W, Wu L-G (2007) Rapid bulk endocytosis and its kinetics of fission pore closure at a central synapse. Proc Natl Acad Sci USA 104:1023410239. 